\title{
Simulating Wind Turbine Interactions using the Vorticity Transport Model
}

\author{
Timothy M. Fletcher* and Richard E. Brown ${ }^{\dagger}$ \\ University of Glasgow, Glasgow, G12 8QQ, United Kingdom
}

\begin{abstract}
The aerodynamic interactions that can occur within a wind farm result in the constituent turbines generating a lower power output than would be possible if each of the turbines were operated in isolation. Tightening of the constraints on the siting of wind farms is likely to increase the scale of the problem in the future. The aerodynamic performance of turbine rotors and the mechanisms that couple the fluid dynamics of multiple rotors can be understood best by simplifying the problem and considering the interaction between only two rotors. The aerodynamic interaction between two rotors in both axial and yawed wind conditions has been simulated using the Vorticity Transport Model. The aerodynamic interaction is a function of the tip speed ratio, the separation between the rotors, and the angle of yaw to the incident wind. The simulations show that the momentum deficit at a turbine operating within the wake developed by the rotor of a second turbine can limit substantially the mean power coefficient that can be developed by the turbine rotor. In addition, the significant unsteadiness in the aerodynamic loading on the rotor blades that results from the inherent asymmetry of the interaction, particularly in certain configurations and wind conditions, has considerable implications for the fatigue life of the blade structure and rotor hub. The Vorticity Transport Model enables the simulation the wake dynamics within wind farms and the subsequent aerodynamic interaction to be evaluated over a broad range of wind farm configurations and operating conditions.
\end{abstract}

\section{Nomenclature}

A rotor disk area

$C_{n} \quad$ force coefficient normal to blade chord

$C_{P} \quad$ rotor power coefficient, $P / \frac{1}{2} \rho A V_{\infty}{ }^{3}$

$C_{t} \quad$ force coefficient tangent to blade chord

$Q \quad$ low speed shaft torque

$R \quad$ rotor radius

$S \quad$ vorticity source

$u \quad$ flow velocity

$u_{b} \quad$ flow velocity relative to blade

$V_{\infty} \quad$ wind velocity

$x_{h}, y_{h}$ downwind and crosswind spacing

$\alpha \quad$ angle of attack

$\begin{array}{ll}\lambda & \text { tip speed ratio, } \Omega R / V_{\infty} \\ \nu & \text { kinematic viscosity } \\ \rho & \text { density } \\ \Omega & \text { rotational speed } \\ \omega & \text { vorticity } \\ \omega_{b} & \text { bound vorticity } \\ \psi & \text { rotor azimuth } \\ \Psi & \text { yaw angle }\end{array}$

Abbreviations
Ref reference rotor
DW $\quad$ downwind rotor

\section{Introduction}

The aerodynamic interactions between the constituent turbines of a wind farm have been known for some time to degrade its overall power output (see the survey papers by Vermeer, Sørensen and Crespo, ${ }^{1}$ and Crespo, Hernández and Frandsen ${ }^{2}$ ). The interaction is principally a result of the dynamics of the wakes of the turbines and thus may be exacerbated by the configuration of the turbines within the farm. A wake is created downwind of a turbine rotor as a consequence of its generation of an aerodynamic torque. At the

\footnotetext{
*Post-doctoral Research Assistant, Dept. of Aerospace Engineering, AIAA Member.

${ }^{\dagger}$ Mechan Professor of Engineering, Dept. of Aerospace Engineering, AIAA Member.
} 
typical design conditions at which horizontal-axis wind turbines are operated, the wind speed is high enough relative to the tip speed of the rotor for the vortical structures which form its wake to be convected many rotor radii downwind without significant viscous dissipation. Any turbines located downwind that become immersed within this wake will experience a reduction in the energy within their incident flow and thus a reduction in their power output. The separation between the turbines in wind farms can vary significantly, particularly at onshore sites where the size of installations is constrained by the shortage of suitable ground for the turbine foundations. As indicative examples, the turbines in the onshore wind farm at West Kilbride in Scotland are spaced approximately ten rotor radii apart, whilst those at the offshore Horns Rev wind farm in Danish waters are separated by a distance of 14 rotor radii. In practice, the distribution of the turbines within wind farms is designed to minimize, where possible, the interaction between the turbines under prevailing wind conditions.

The major constraints imposed on the overall area which may be occupied by a wind farm, whether onshore or offshore, include, for example, the topography of the chosen site and the need not to impose unduly on shipping lanes. Significant interest in understanding the aerodynamic interactions within wind farms in order to allow the optimization of wind farm configurations and to permit increases in turbine density has developed over the last 30 years. Newman ${ }^{3}$ used a theoretical approach that accounted for different types of surface topography to show that significant power losses would occur if the turbine spacing was too low. In 1978, Builtjes and $\mathrm{Smit}^{4}$ investigated the optimal spacing of vertical-axis wind turbines using a combination of wind tunnel data and numerical methods, indicating that as the number of turbines within the farm is increased, so should the spacing between the turbines in order to achieve optimal power output. Lissaman ${ }^{5}$ developed a numerical model to predict the power degradation in wind turbine arrays using momentum conservation and reduced-order models for wake growth and ground effect. Milborrow ${ }^{6}$ used a combination of experimental and analytical methods to investigate the effects of interaction between wind turbines on the overall efficiency of a farm. It was estimated that the power output from a typical wind farm, as envisaged at that time, could be $25 \%$ lower than that of an equivalent number of turbines operating in isolation.

Sforza, Sheerin and Smorto ${ }^{7}$ developed a theoretical model in which the wakes induced by the wind turbine rotors and also the turbulent atmospheric boundary layer were treated as small perturbations to a uniform freestream. Results from this model were then compared with the velocity measured at various downwind locations during wind tunnel tests. Sforza, Sheerin and Smorto found good agreement between theoretical predictions of the velocity deficit downwind of a given turbine rotor and experimental measurements. In addition to experimental studies of the wakes of individual turbines, wind tunnel tests have been performed on arrays of sub-scale model turbines, for example by Ainslie, Hassan, Parkinson and Taylor in $1990 .^{8}$ Voutsinas, Rados and Zervos ${ }^{9}$ developed an effective means of modeling the annual energy output of arrays of large numbers of turbines using the Abramovich theory of turbulent jets. Voutsinas and his co-workers concluded that the simplified modeling of the individual turbines in this case was not able to fully represent all of the pertinent physics in the development of the flow, and that to understand better the energy extraction process, a more physically realistic representation of the wake of each wind turbine would be required. Most recently, the pan-European UpWind project ${ }^{10}$ has been conducted to improve the modeling of the flow within large offshore wind farms, principally using conventional CFD methods, with the aim of improving power predictions.

In this paper, a computational model which solves the incompressible Navier-Stokes equation in vorticityvelocity form will be introduced, and then applied to a series of simplified wind farm interactions. By adopting this approach, some of the inherent problems of numerical dissipation that are encountered when solving the governing equations of fluid motion in pressure-velocity-density form may be avoided. By providing a highfidelity representation of the structure and evolution of the wake of a wind turbine rotor and its interaction with other turbines within a wind farm, the fluid dynamics associated with the power losses discussed above can be better understood. Importantly, this may allow the designers of wind farms to explore ways in which to alleviate the adverse effects of interaction, including not only power losses, but also the unsteady loads that can result in a reduction in the fatigue life of the components within the hub and transmission system. Any strategy for the active optimization of wind farm performance using individual turbine control, or passive alleviation of wake effects, will require a detailed characterization of the velocity that is induced at the blades of each individual turbine by the wakes of other turbines. The approach that is presented in this paper, through providing an accurate and detailed model of the flow physics that are associated with the interactions between individual turbines, may contribute to the development of effective strategies for 
control of the yaw angle of the turbine, the feather of the blades, and, in the future, perhaps also the twist distribution along the blades, in order to allow the power losses within wind farms to be mitigated.

\section{Computational Aerodynamics}

The aerodynamic performance of two generic horizontal-axis wind turbine rotors and their fluid dynamic interaction has been simulated in a number of representative configurations using the Vorticity Transport Model (VTM) developed by Brown, ${ }^{11}$ and extended by Brown and Line. ${ }^{12}$ The VTM enables the simulation of wind turbine aerodynamics and performance using a high-fidelity model of the wake generated by the turbine rotor. The time-dependent Navier-Stokes equation is discretized in finite-volume form using a structured Cartesian mesh within the domain surrounding the turbine rotor. After making the physically realistic assumption of incompressibility within the wake, the Navier-Stokes equations are cast into the vorticity-velocity form

$$
\frac{\partial}{\partial t} \omega+u \cdot \nabla \omega-\omega \cdot \nabla u=S+\nu \nabla^{2} \omega
$$

The advection, stretching, and diffusion terms within the vorticity transport equation describe the changes in the vorticity field, $\omega$, with time at any point in space, as a function of the velocity field, $u$, and the viscosity, $\nu$. The physical condition that vorticity may neither be created nor destroyed within the flow, and thus may only be created at the solid surfaces immersed within the fluid, is accounted for using the vorticity source term, $S$. The vorticity source term is determined as the sum of the temporal and spatial variations in the bound vorticity, $\omega_{b}$, on the turbine blades:

$$
S=-\frac{d}{d t} \omega_{b}+u_{b} \nabla \cdot \omega_{b}
$$

The bound vorticity distribution on the blades of the rotor is modeled using an extension of the Weissinger-L lifting-line theory. ${ }^{13}$ The velocity field is related to the vorticity field by using a Cartesian fast multipole method to invert the differential form of the Biot-Savart law:

$$
\nabla^{2} u=-\nabla \times \omega
$$

Use of the fast multipole method, in conjunction with an adaptive grid in which cells are only present within the calculation when the vorticity within them is non-zero, dramatically increases the computational efficiency of the scheme when compared to an equivalent calculation performed on a fixed grid. In the current analysis, the ground plane is not modeled and therefore the velocity gradient associated with the atmospheric boundary layer does not influence the flow field surrounding the turbines. The method is rendered effectively boundary-free as cells may be created, when necessary, on a Cartesian stencil which extends to infinity, using the assumption that there is zero vorticity outside the wake. ${ }^{12}$ An assumption is made that the Reynolds number within the computational domain is sufficiently high that the governing flow equation may be solved in inviscid form. Dissipation of the wake does still occur, however, through the mechanism of natural vortical instability. The numerical diffusion of vorticity within the flow field surrounding the wind turbines is kept at a very low level by using a Riemann problem technique based on the Weighted Average Flux method developed by Toro ${ }^{14}$ to advance equation 1 through time. This approach allows highly efficient multi-rotor simulations, and permits many rotor revolutions to be captured without significant spatial smearing of the wake structure, in contrast to the performance of more conventional CFD techniques based on the pressurevelocity-density formulation of the Navier-Stokes equations.

The VTM was originally developed for simulating the flow field surrounding helicopters, but is an aerodynamic tool which is highly suitable for application to the study of wind turbine rotors. The VTM has been used successfully to investigate the aerodynamic interaction between the main and tail rotors of a helicopter, ${ }^{15}$ and for the study of helicopter main rotor - fuselage interaction. ${ }^{16}$ It has also been used to model the performance of rotors in axial flight ${ }^{17}$ and to examine the fluid dynamics of the rotor vortex ring state. ${ }^{18}$ Notably, it has also been validated against experimental data for co-axial helicopter rotors. ${ }^{19}$ 


\section{Wind Turbine Model}

The rotor model that was used for the present study is a three-bladed version of the rotor used for Phase VI of the NREL Unsteady Aerodynamics Experiment. ${ }^{20}$ Three blades were used in order to increase the solidity of the rotor and thus to increase the rotor power coefficient at lower tip speed ratios, such that the peak performance of the rotor occurred at tip speed ratios in the range 6-8. The structural deformation of the blades, in the form of bending, torsion and extension, are not modeled in the current analysis, and the blades are rigidly attached to the hub. In addition, the aerodynamic effects associated with the hub assembly and tower have not been modeled. The pitch of the blades and rotational speed of each of the rotors is constant, such that the downwind rotor does not compensate in any way for the reduction in torque available as a result of interaction. Whilst this may not be entirely representative of turbine operation in practice, it is essential first to understand the fluid dynamics associated with wake impingement before adding the complication associated with the turbine control system. For a detailed description of the blade geometry see Ref. 20; the key properties of the turbine rotors are summarized in table 1, however.

Table 1. Rotor data.

\begin{tabular}{lc}
\hline Type of rotor & rigid \\
No. of blades & 3 \\
Rotor radius & $\mathrm{R}$ \\
Airfoil & NREL S809 \\
Rotational speed & $\Omega$ (constant) \\
Blade tip pitch & $3^{\circ}$
\end{tabular}

Table 2. Simulated configurations and operating conditions.

\begin{tabular}{cccc}
$\lambda$ & $x_{h}$ & $y_{h}$ & $\Psi$ \\
\hline 6 & $4 \mathrm{R}$ & 0 & $0^{\circ}$ \\
6 & $4 \mathrm{R}$ (reverse rotation) & 0 & $0^{\circ}$ \\
6 & $8 \mathrm{R}$ & 0 & $0^{\circ}$ \\
6 & $12 \mathrm{R}$ & 0 & $0^{\circ}$ \\
8 & $4 \mathrm{R}$ & 0 & $0^{\circ}$ \\
8 & $8 \mathrm{R}$ & 0 & $0^{\circ}$ \\
8 & $12 \mathrm{R}$ & 0 & $0^{\circ}$ \\
8 & $4 \mathrm{R}$ & $4 \mathrm{R}$ & $15^{\circ}$ \\
8 & $4 \mathrm{R}$ & $4 \mathrm{R}$ & $30^{\circ}$ \\
8 & $4 \mathrm{R}$ & $4 \mathrm{R}$ & $45^{\circ}$
\end{tabular}

The effect of the wake created by a reference rotor on the performance of a second turbine rotor placed downwind has been investigated over a range of rotor separations and different wind conditions. The rotor system is simulated in axial and in yawed wind conditions at tip speed ratios of six and eight. In axial flow, three different values for the downwind separation between the rotor were used: four, eight and twelve rotor radii, according to the conventions defined in figure 1. In yawed wind conditions, both the downwind and the crosswind separations between the rotors were fixed at four rotor radii whilst angles of yaw to the wind of $15^{\circ}, 30^{\circ}$, and $45^{\circ}$ were simulated. In addition, a simulation was performed in which the downwind rotor rotates in the opposite sense to the reference rotor. In this simulation, the rotors were separated by four rotor radii and subjected to an axial wind giving a tip speed ratio of six. A list of the configurations that were simulated is given in table 2 .

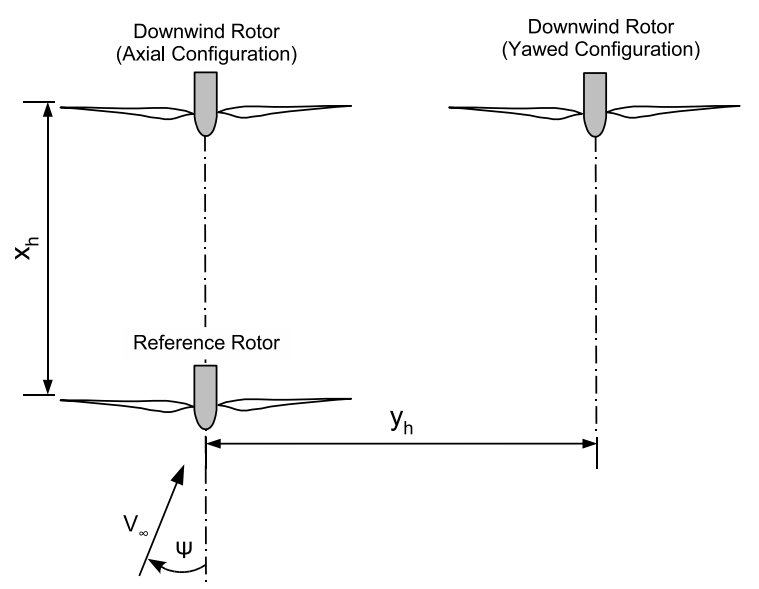

Figure 1. Relative locations of simulated wind turbine rotors. 


\section{Verification of Aerodynamic Predictions}

Before investigating the effect of aerodynamic interaction on the performance of horizontal-axis wind turbines, it is essential to first establish that the requisite fluid dynamic features within the flow surrounding the turbine rotor, and hence the aerodynamic loads on the blades of the rotor, are correctly simulated by the VTM. Figure 2 shows comparisons of the normal force coefficient and the shaft torque predicted by the VTM and equivalent data obtained during Phase VI of the NREL Unsteady Aerodynamics Experiment. ${ }^{20}$ The rotor tested during this experiment was a two-bladed version of the rotor described in Section III. The rotor was mounted upwind of the tower, and operated in an axial flow condition at a wind speed of $7 \mathrm{~m} / \mathrm{s}$ (translating into a tip speed ratio of 5.4). The data presented in figure 2(a) represent the mean values of normal force coefficient over one complete revolution with the rotor operating at an approximately constant rotational speed and therefore in a quasi-steady state. The error bars attached to the experimental data points indicate the variability in the normal force coefficient at each radial location over the duration of the particular Phase VI test replicated here.

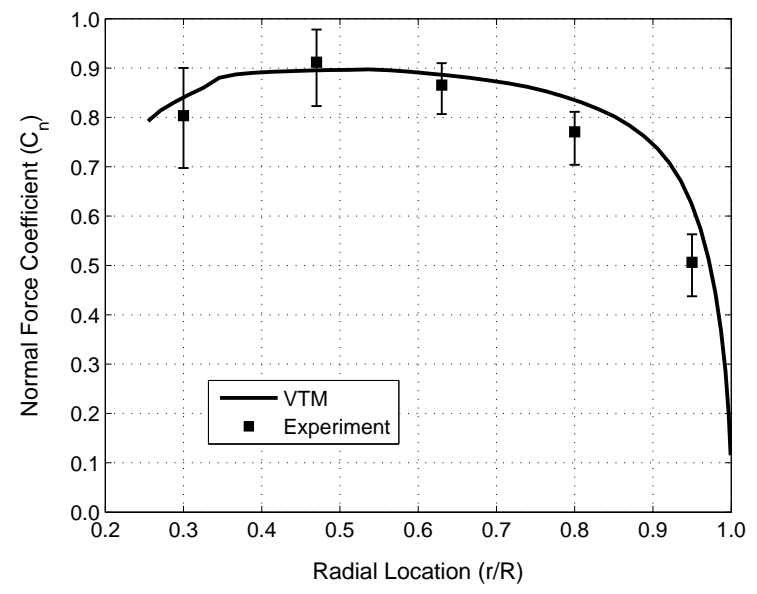

a) Radial distribution of normal force coefficient.

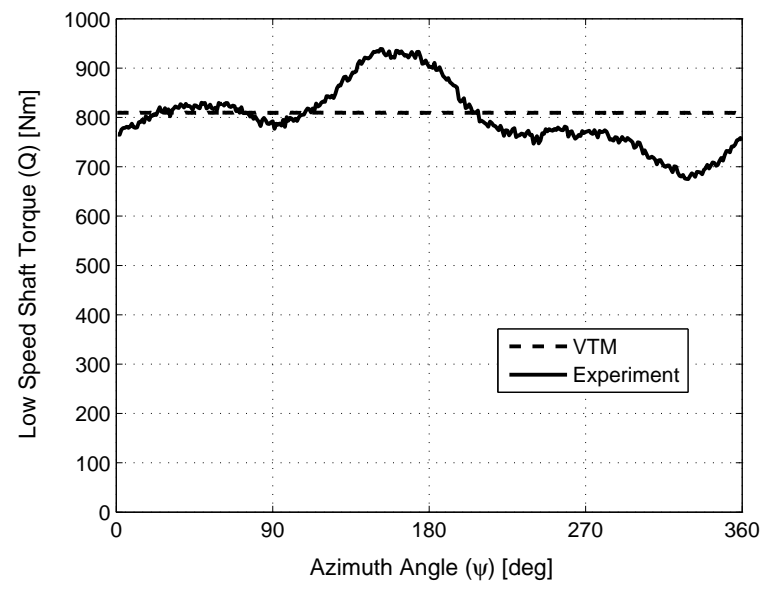

b) Azimuthal variation of shaft torque.

Figure 2. HAWT aerodynamic performance, computed using the VTM, compared with data from Phase VI of the NREL Unsteady Aerodynamics Experiment; $\lambda=\mathbf{5 . 4}$.

Figure 2(a) shows that the magnitude of the normal force coefficient along most of the blade is predicted well by the VTM. The normal force coefficient is slightly over-predicted along the outboard third of the blade. The results predicted using the VTM are, however, satisfactory given the simplicity of the blade aerodynamic model. The variation in the experimental measurements, along with the limitations placed on predicting the centrifugal effects within the boundary layer inherent in the quasi-two-dimensional model of the blade aerodynamics, may easily combine to compensate for this modest disparity. Of most relevance to the current work is the accuracy with which the VTM can predict the torque delivered to the shaft of the turbine rotor. In figure 2(b) it can be observed that, whilst the VTM does not replicate the unsteadiness in the shaft torque with the rotation of the rotor, the mean torque is very well predicted. Indeed, if the experimental data is averaged over the complete revolution shown, the magnitude of the low speed shaft torque collapses almost exactly onto the steady value that is computed using the VTM.

The model of the blade aerodynamics that is implemented within the Vorticity Transport Model uses a look-up table, based on local angle of attack, to obtain the airfoil lift, drag, and pitching moment at each blade section. The best predictions of the aerodynamic loading on the wind turbine blades are thus obtained when the flow is almost entirely attached and is aligned with the chord of the blade, in other words when the angle of attack at each blade section is sufficiently low that the blade operates within the linear aerodynamic regime. The VTM is thus known to provide the most accurate predictions of the overall loading on the turbine and its shaft torque at high tip speed ratio. It is for this reason that tip speed ratios near the higher end of the realistic range for the type of rotor that was simulated were selected for examination in the present study. 


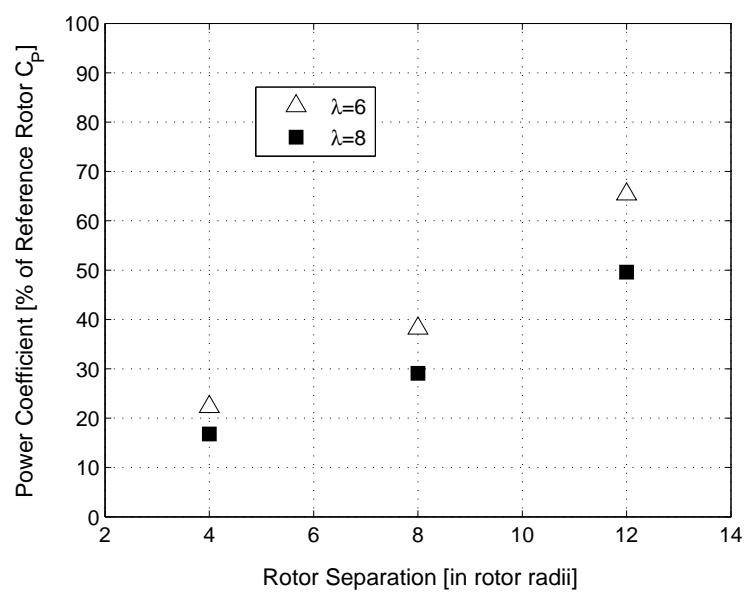

Figure 3. Power coefficient of the downwind turbine (as a percentage of the power coefficient of the reference rotor) as a function of the separation between the rotors for two different tip speed ratios of the reference rotor.

\section{Interaction in Axial Wind Conditions}

Given the inherent symmetries within the system, the basic effects of aerodynamic interaction between two rotors are most readily exposed in a study of the couplings within a system in which the reference and downwind rotors are axially co-aligned and the incident wind is parallel to the shared axis of rotation of the turbines.

\section{V.A. Rotor Performance}

The generation of lift and drag forces on the rotor blades results in a momentum deficit within the wake that develops downwind of the turbine. When a second turbine is subject to direct impingement by the wake of an upwind rotor, then the effective wind velocity that it experiences is reduced compared to that incident on the upwind rotor. The reduced flow speed manifests as a reduction in the power that may be extracted from the wind by the downwind turbine.

Figure 3 shows VTM predictions of the power coefficient developed by turbine rotors located at various distances downwind of a reference rotor, and demonstrates that the reduction in the mean power coefficient of the downwind rotor is strongly dependent on the wind speed and on the separation between the two rotors. When the reference rotor operates at a tip speed ratio of six, the rotor positioned four rotor radii downwind develops only $22 \%$ of the power coefficient of the reference rotor. As the spacing between the rotors is increased, the power coefficient of the downwind rotor recovers in a highly nonlinear fashion as the adverse effect of the interaction between the rotors is reduced. If the tip speed ratio of the reference rotor is increased to eight (equivalent to a reduction in wind speed) then the aerodynamic performance of the downwind rotor is degraded further, confirming expectations that the adverse effects of aerodynamic interaction should increase in severity at lower wind speeds. The recovery in the power coefficient of the downwind rotor with increased separation between the rotors follows a very similar trend at both of the tip speed ratios that have been simulated, suggesting that a simple semi-empirical model may be capable of reproducing the variation of power output with turbine separation shown in figure 3. Development of this model is complicated though by its reliance on a reduced-order theory to describe the downstream variation of the mean velocity within the turbine wake, particuarly in the presence of the vortex instabilities that will be shown in the following section of the paper to have a key influence on the performance of the downwind turbine.

Figures 4(a) and 4(b) show the variation in the power coefficient developed by the reference and downwind rotors operating in axial wind conditions at tip speed ratios of six and eight, respectively, over the entire time period over which the each of the rotors have been operated following start-up. The power coefficient of both the reference and downwind rotors undergoes a transient overshoot following the impulsive start-up of the rotor at the beginning of each simulation. Once the initial transient loads have subsided, the reference rotor continues to develop an almost constant power coefficient as a result of its operation within a uniform 


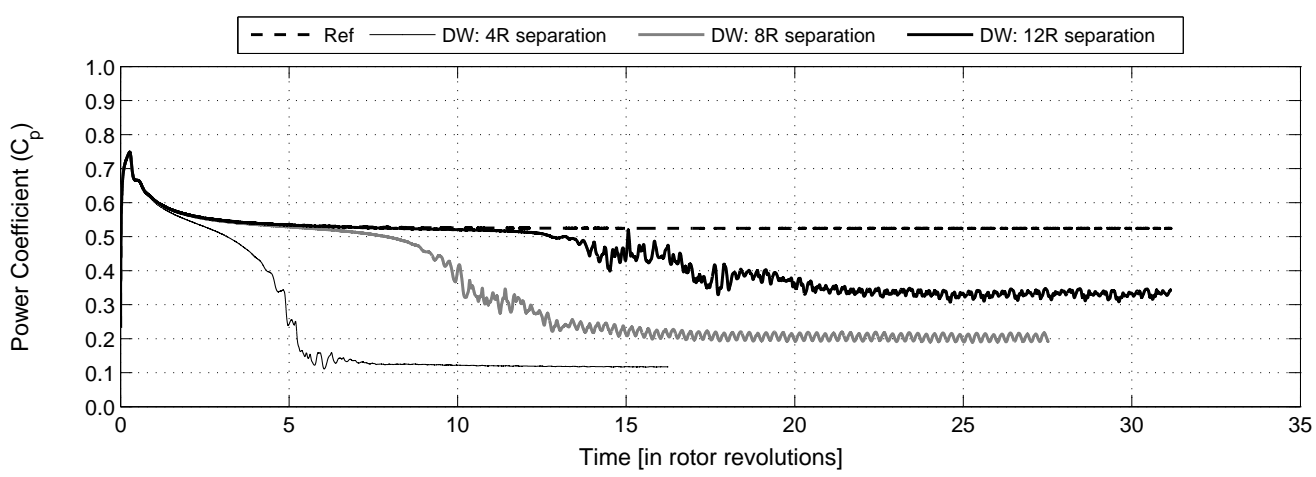

(a) $\lambda=6$

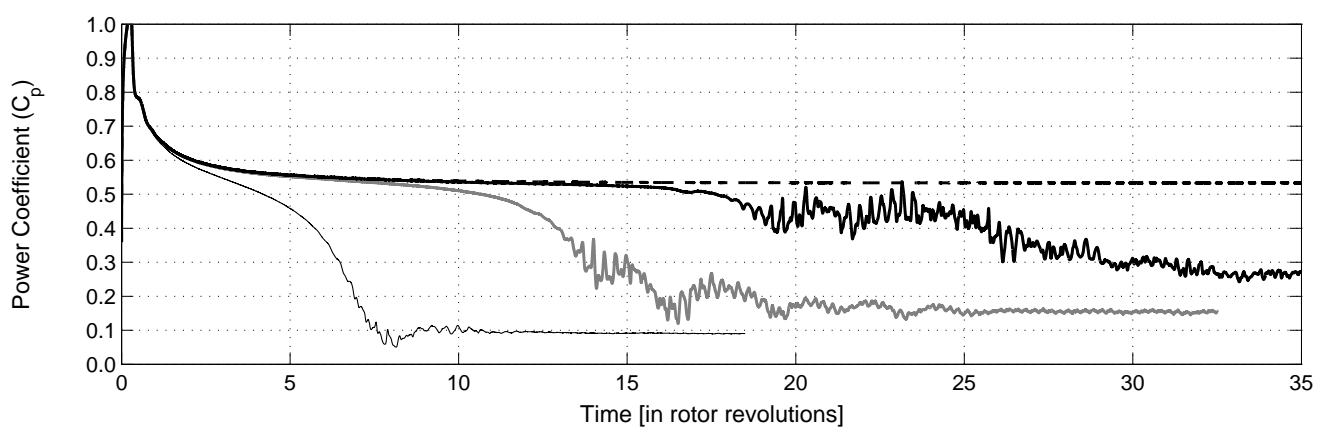

(b) $\lambda=8$

Figure 4. Reduction in power coefficient as a function of time after an instantaneous start, for rotors located 4, 8, and 12 rotor radii downstream of the reference rotor (Ref: reference rotor, DW: downwind rotor).

and steady incident wind, unperturbed by impingement from the wake of other rotors. The separation between the reference and downwind rotors is sufficiently large that the aerodynamic performance of the reference rotor is not affected by the presence of the downwind rotor. The initial impingement of the wake of the reference rotor results in a period of time during which the power absorbed by the downwind rotor fluctuates considerably. The transient unsteadiness in the power developed by the downwind rotor is more persistent the greater the spacing between the reference and downwind rotors, as can be seen by comparing the variations in power coefficient presented in figures $4(\mathrm{a})$ and $4(\mathrm{~b})$.

The reduction in the power coefficient developed by the downwind rotor is, of course, the result of this rotor developing a driving torque which is lower than that developed by the reference rotor. Figures 5(a) and 5(b) show the radial distribution of normal force coefficient, at tip speed ratios of six and eight respectively, developed by the rotors represented in figure 3. It is clear that the power coefficient developed by a rotor operating within the wake of another is reduced as a result of its blades developing an aerodynamic loading which is significantly lower than that which is generated on the blades of the rotor when operated in isolation. Indeed, the aerodynamic performance of the blades of the downwind rotor is lower than that of the reference rotor, along the entire length of the blades. This indicates that the downwind rotor as a whole is adversely affected by aerodynamic interaction with the wake of the reference rotor, as is to be expected given the geometry of the wake. When the reference and downwind rotors are separated by only four or eight rotor radii, the reduction in normal force coefficient along the length of the blades is proportionately larger when those rotors operate at a higher tip speed ratio.

The reduction in the mean power coefficient of the downwind rotor is largest when the spacing between the downwind and reference rotors is smallest. However, the unsteadiness in the power coefficient of the downwind rotor is greatest when the rotors are separated by the largest distance, as shown in figures 4(a) and $4(\mathrm{~b})$. The variation in the normal force coefficient along the blades of the downwind rotor over the duration of one rotor revolution is indicated using error bars in figure $5(\mathrm{~b})$, for the case where the separation between the rotors is twelve rotor radii and the unsteadiness in the power coefficient was largest. It is evident from figure 5(b) that the relatively low amplitude of the fluctuations in the normal force coefficient 


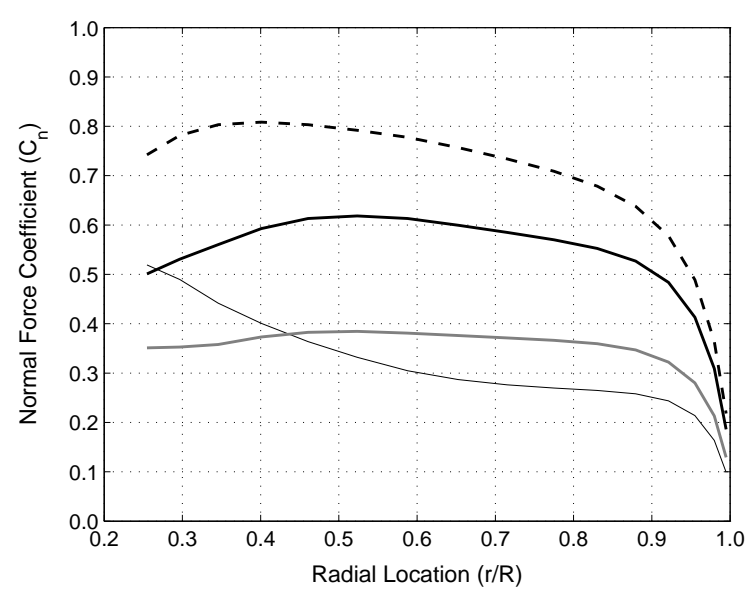

a) Radial distribution of normal force coefficient; $\lambda=6$.

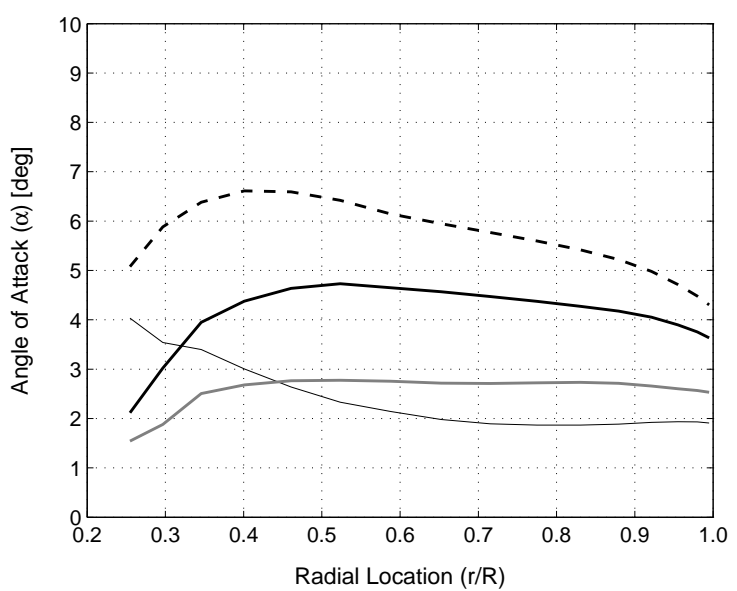

c) Radial distribution of angle of attack; $\lambda=6$.

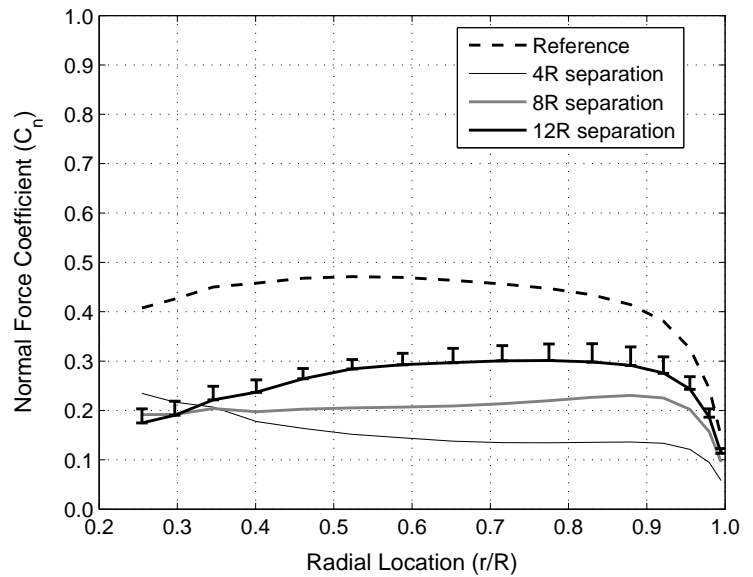

b) Radial distribution of normal force coefficient; $\lambda=8$.

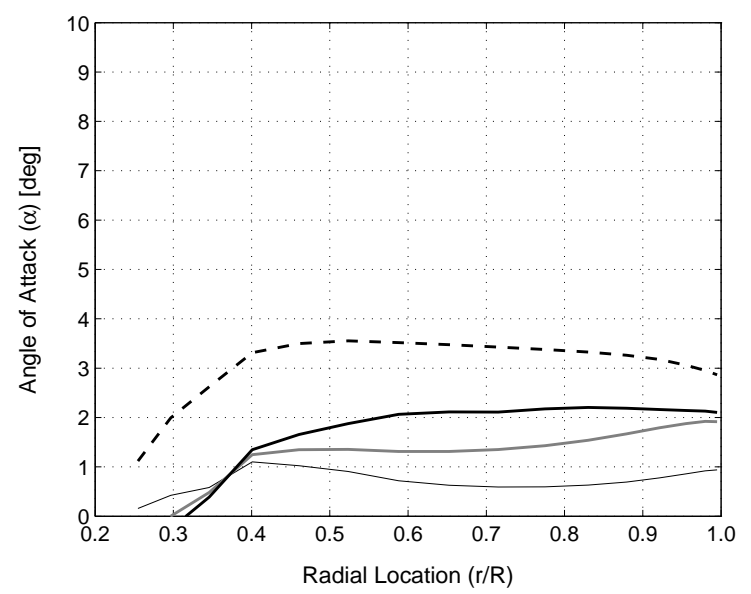

d) Radial distribution of angle of attack; $\lambda=8$.

Figure 5. Variation in the aerodynamic loading on axially co-aligned rotors, operating in axial flow conditions, as a function of the separation between the rotors for two different tip speed ratios of the reference rotor.

that occur over the duration of each revolution confirm the recurrence of the trends indicated in figure 5 . Indeed, the amplitude of fluctuations in both the normal force coefficient and the angle of attack were found to be lower in each of the other configurations that were simulated. The distributions of normal force shown in figures 5(a) and 5(b) are consistent with the variation of angle of attack along the length of the blades shown in figures $5(\mathrm{c})$ and $5(\mathrm{~d})$. The relative reduction in the angle of attack along the blades with the separation between the rotors is larger at the higher of the two tip speed ratios, as is shown by a comparison of figures $5(\mathrm{c})$ and $5(\mathrm{~d})$.

\section{V.B. Structure of the Flow Field}

The aerodynamic performance of a rotor positioned downwind of a second rotor is strongly influenced by the wake of the upwind rotor. Indeed, the effect on the power coefficient of the downwind rotor that is demonstrated in figure 3 indicates the substantial influence of the velocity induced by the wake of the rotor system. Figure 6 shows the characteristic features of the wake system surrounding the reference and downwind rotors for three rotor separations $(4 \mathrm{R}, 8 \mathrm{R}$, and $12 \mathrm{R})$ and with the reference rotor operating at a tip speed ratio of eight. To the left of each sub-figure is a instantaneous snapshot of the three-dimensional wake structure. The wake is represented by plotting a surface of constant vorticity magnitude in the flow. To the right of each sub-figure, a contour map is plotted showing the distribution of flow speed (velocity magnitude) within the wake system. 


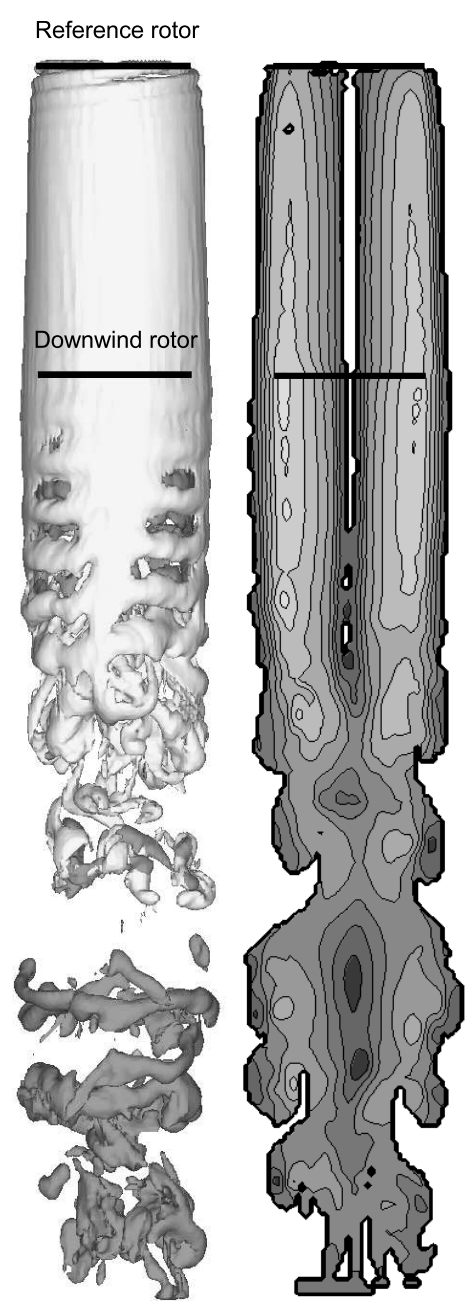

a) rotor separation $4 \mathrm{R}$

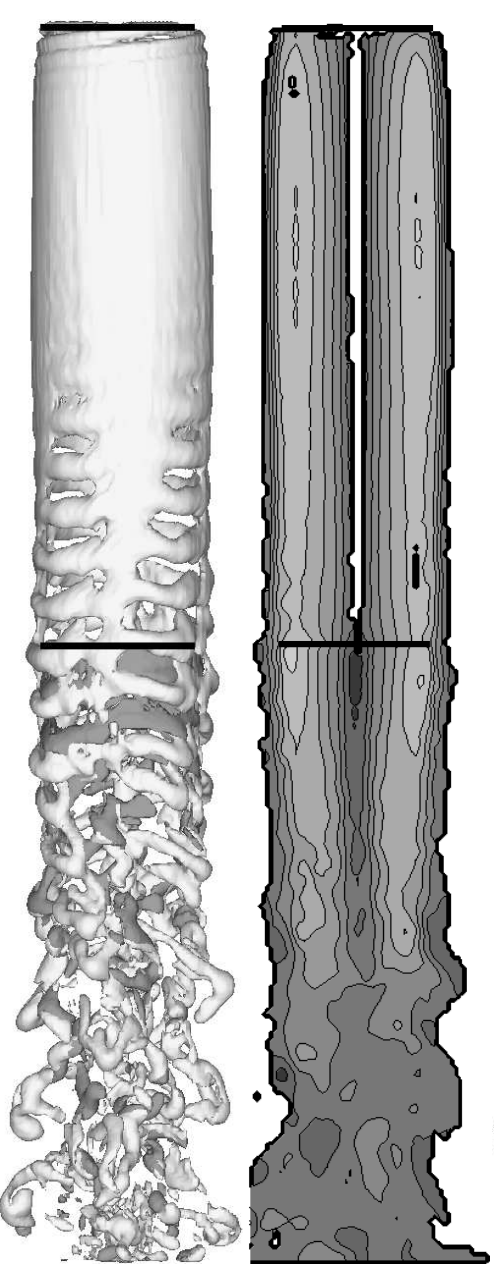

b) rotor separation $8 \mathrm{R}$

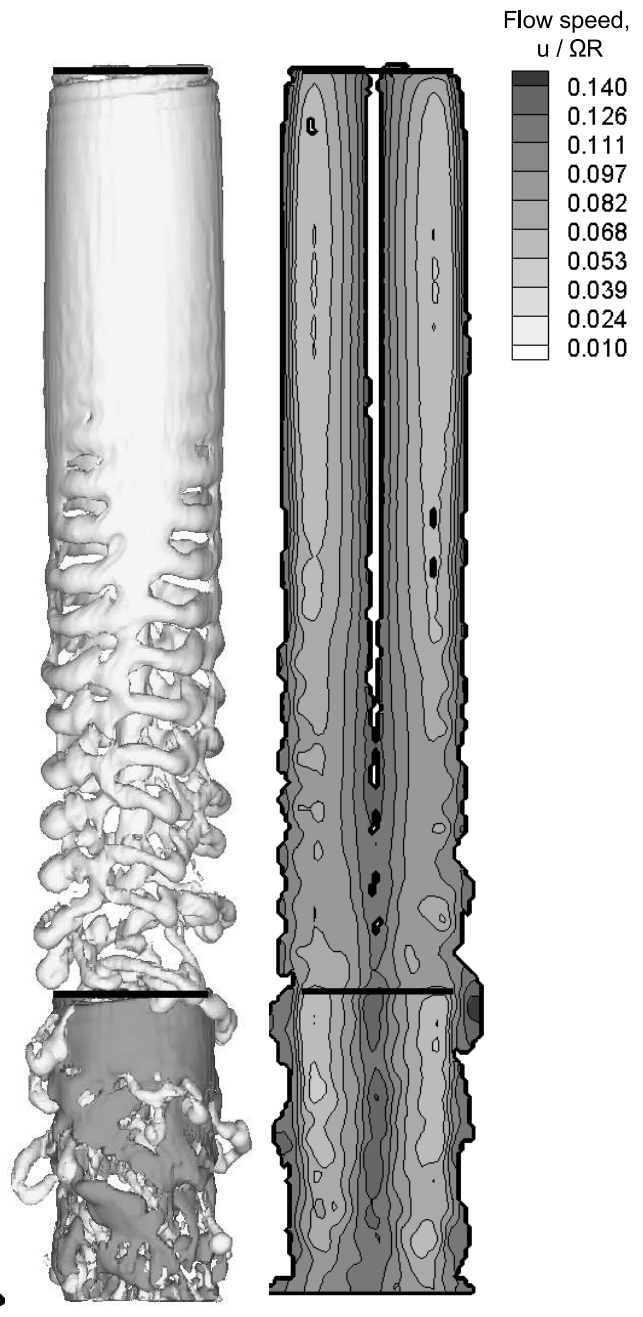

c) rotor separation $12 R$

Figure 6. Vorticity and velocity distributions in the flow surrounding two axially co-aligned rotors; $\lambda=8$ for the reference rotor. At left of each sub-figure: instantaneous iso-surface of vorticity representing the wake (light gray: reference rotor, dark gray: downwind rotor). At right of each sub-figure: contours of flow speed normalized using the rotor tip speed.

Figure 6 shows that the helical structure developed by the tip vortices from a rotor operating at a low tip speed ratio persists for a relatively limited distance downwind of the rotor. The natural instabilities within the wake, that manifest first as local pairing and leap-frogging of the vortex filaments, develop with time to form larger aperiodic vortex structures. The impingement of the wake created by the reference rotor on the downwind rotor further promotes the development of disturbances within the wake, augmenting those already present within the wake of the reference rotor. This leads to the breakdown of the structure of the combined wake system of the two rotors, as shown in figure 6(a). The relatively light loading on the blades of the downwind rotor when positioned four rotor radii downwind of the reference rotor necessarily limits the vorticity trailed from that rotor into the wake. As the spacing between the rotors is increased to twelve rotor radii, a coherent and quasi-periodic vortical structure is able to persist to a greater distance downwind of the reference rotor before being disrupted by the impingement of the wake on the downwind turbine, as is indicated by a comparison of the instantaneous snapshots of vorticity shown in figures $6(\mathrm{a})$ and 6 (c). If the tip speed ratio at which the rotor operates is lower (equivalent to a higher wind speed), the helical structure formed by the concentrated tip vortices persists to greater distances downwind since in this case the wake is convected more rapidly by the freestream component of the flow.

Figure 6 illustrates why a re-formulation of blade element momentum theory (BEMT), as has been suggested in some quarters, in order to model the performance of the downwind rotor within the wake of 


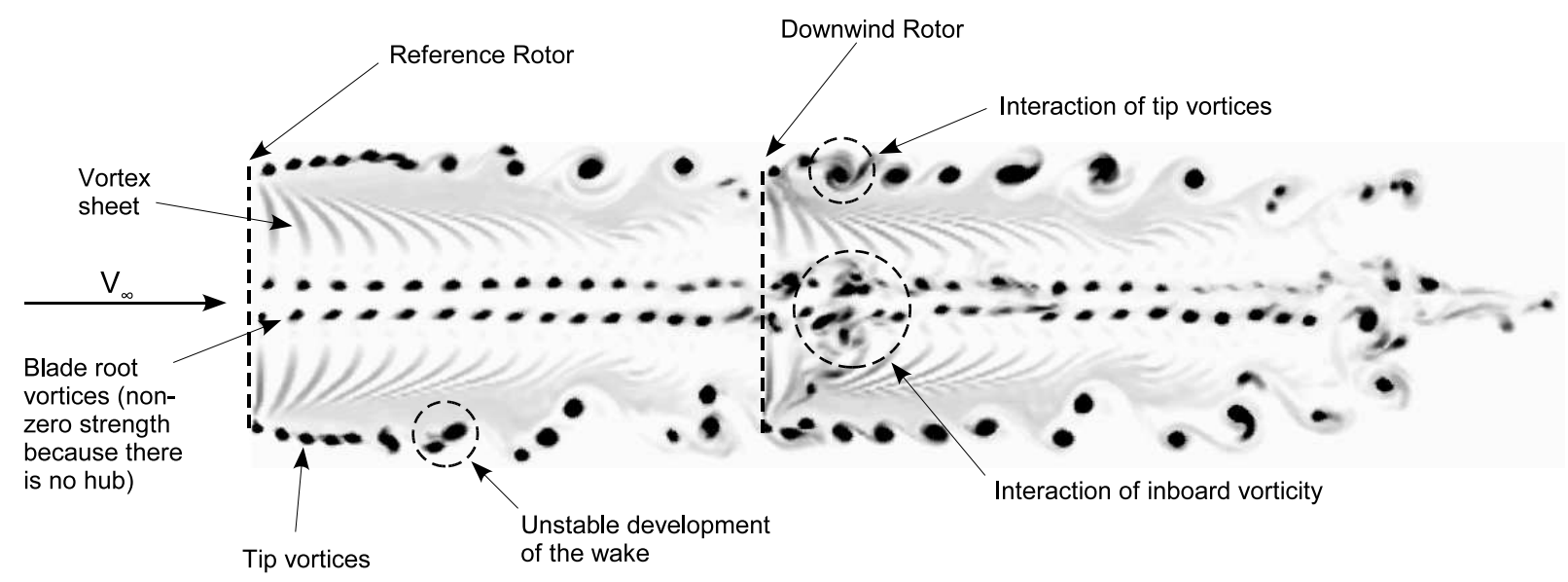

Figure 7. Contours of vorticity magnitude on a plane through the reference and downwind rotors in axial wind conditions (spacing of 4 rotor radii; $\lambda=7$ ).

the reference rotor would be inappropriate, especially where a substantial distance exists between the two rotors. Versions of the BEMT have been developed for co-axial helicopter rotors operating in hover, and the temptation might arise to formulate a co-axial wind turbine BEMT for the windmill brake state. If a similar approach was applied to the aerodynamic interaction between two wind turbine rotors, the power deficiency of the downwind rotor would asymptote to a constant value as the separation between the rotors is increased and hence as the downwind rotor is moved into the fully-expanded portion of the idealized wake. In practice, the separation between the turbines in wind farms is sufficiently large that the aerodynamic performance of the downwind rotor would become insensitive to the separation between the reference and downwind rotors. This result would clearly be at odds with the variation in power coefficient that is shown in figure 3 and the distribution of flow speed within the wake downwind of the reference rotor that is demonstrated by a comparison of figures $6(\mathrm{a}), 6(\mathrm{~b})$, and $6(\mathrm{c})$.

The instantaneous snapshots of the wakes of the reference and downwind rotors, shown in figure 6 , were calculated using a relatively coarse computational grid. In order to examine the vortical structures within the flow field near to the rotors, it is necessary to visualize the wake of the rotor system at significantly finer scales. This is achieved by simulating the flow around the rotor system using a significantly higher density of cells within the computational domain. Figure 7 shows the interaction between the wakes developed from both the reference and downwind rotors. In order to be representative of the fluid dynamics within the wakes of the configurations simulated in this paper, the rotors shown in figure 7 are separated by a distance of four rotor radii and operate at a tip speed ratio of seven. The individual trailed vortices that form downwind of the roots and the tips of the blades, along with the sheet of vorticity that is shed from the trailing edge of each blade, can be observed clearly. At a distance of approximately 1-2 radii downwind of the upstream rotor, vortex pairing and leap-frogging instabilities develop in the tip vortices and result in an aperiodic wake structure by the time the vorticity reaches the downwind rotor. As the vortices that are trailed from the roots and tips of the blades of the upstream rotor impinge on the downwind rotor, they interfere with the development of the helical wake structure of the downwind rotor. Figures 6 and 7 demonstrate the significant effect of both the stability and the dynamics of the wake of the reference rotor on the performance of any rotor located downwind. Indeed, it is clear that the aerodynamic coupling between the rotors in a wind farm is a largely inviscid phenomenon. The natural instability of the vortices within the wake, and the extent to which there is a breakdown in the structure of the wake, largely dictates the velocity deficit at the downwind rotor. The results presented here suggest that the viscous dissipation that occurs at very fine scales within the wake may indeed have only a relatively minor influence on the performance of the downwind rotor.

\section{Interaction during Yawed Operation}

The majority of the aerodynamic interactions within wind farms occur when the turbines are operating 'off-design'. This follows from the practice of designing the orientation and spacing of the turbines within a farm to minimize interactions when the wind is blowing in one specific direction, invariably taken to be the 
prevailing wind direction. The ideal response to changes in wind direction would be to rotate each turbine rotor into the wind, assuming that the wind speed remains below the threshold at which the rotor needs be parked. In practice, however, it is inevitable that a wind turbine will operate at some angle of yaw to the incident wind, and therefore it is highly appropriate to study the effects of aerodynamic interaction under yawed conditions.

Figure 8 shows the power coefficient developed by both the reference and downwind rotors when operating at three different angles of yaw to the incident wind. In each case, as with figure 4 shown earlier, the data is presented over the complete period of operation from the initial starting of the rotor to the point at which the downwind rotor operates at a quasi-steady mean power coefficient. Indeed, it is worth noting that the short distance between the reference and downwind rotors results in the onset of interaction between the rotors after only a few rotor revolutions. Figure 8 demonstrates that, for small angles of yaw, there is a modest increase in the power coefficient of the downwind rotor as a result of the aerodynamic interaction with the reference rotor, rather than the reduction in performance that may be expected given the reduction in the power coefficient of the downwind rotor in each of the other configurations presented in this paper. The reason for this seeming anomaly is revealed in figure 9 (a) which illustrates the instantaneous wake structure of the reference and downwind rotors in the case where the angle of yaw is $15^{\circ}$. The most substantial vortical structures within the wake of the reference rotor follow a trajectory which precludes their direct impingement on the downwind rotor. Nevertheless, the wake of the reference rotor can be seen to be deformed modestly as it passes the downwind rotor, indicating the presence of aerodynamic coupling between the two rotors even in this case. In this configuration, the wake of the upwind rotor is found to induce a mild increase in the local wind speed at the downwind rotor, yielding the increase in its power coefficient that is shown in figure 8 .

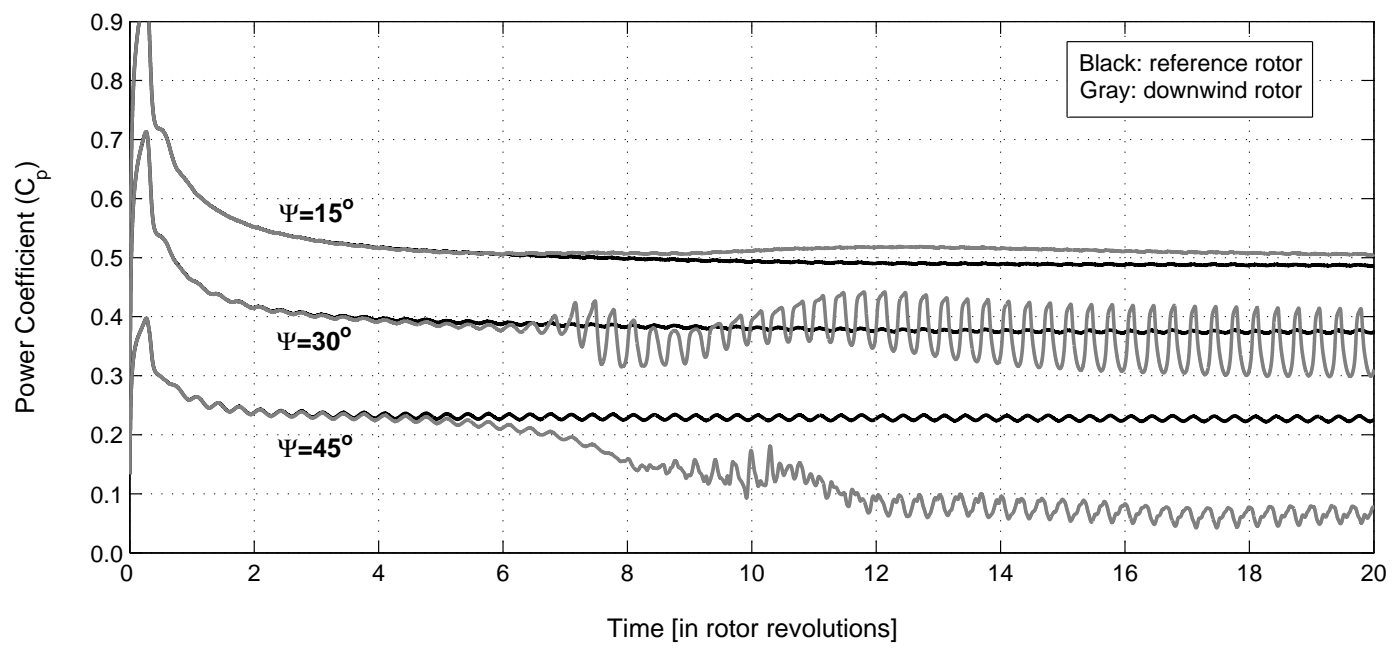

Figure 8. Reduction in power coefficient of the downwind rotor as a result of aerodynamic interaction for yaw angles of $15^{\circ}, 30^{\circ}$, and $45^{\circ}$.

When a wind turbine rotor operates at a non-zero angle of yaw to the incident wind an asymmetry develops in the loading on the rotor. This asymmetry in loading occurs as effective advancing and retreating sides are established on the rotor disk, in which the translational velocity of the blades is orientated toward, or away from, the component of the incident wind in the plane of the disk, respectively. The asymmetry in loading on the rotor disk results in fluctuations in the power coefficient at the blade passage frequency (in this case $3 / \mathrm{rev}$ ), as shown in figure 8 . If the incident wind makes only a modest angle of yaw to the turbine rotors, such as, for example, in the case of a $15^{\circ}$ angle of yaw as shown in figure 8 , the amplitude of the unsteady component of the power developed by the reference rotor is small compared with the mean power. When the angle of yaw of the turbine rotors is such that there is partial impingement of the wake of the reference rotor on the downwind rotor, for example when the rotors operate at a $30^{\circ}$ angle of yaw, only the amplitude of the unsteady component of the power developed by the downwind rotor increases significantly. This increase in unsteady loading is caused by the impingement of the wake developed by the reference rotor on only one side of the disk of the downwind rotor, as shown in figure $9(\mathrm{~b})$. 


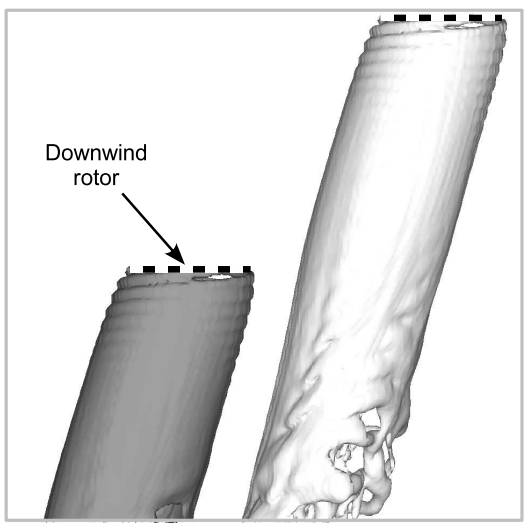

a) Yaw angle $=15^{\circ}$

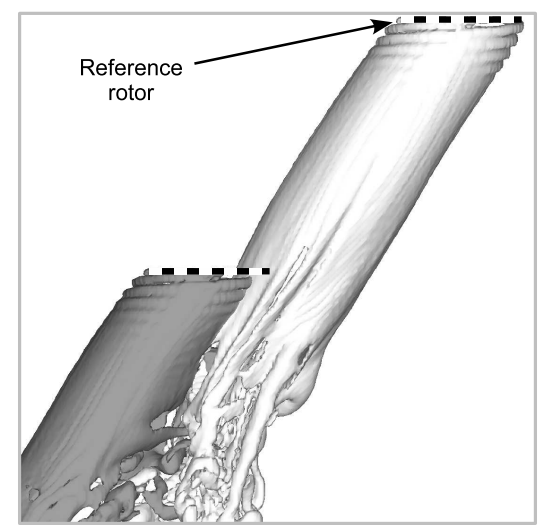

b) Yaw angle $=30^{\circ}$

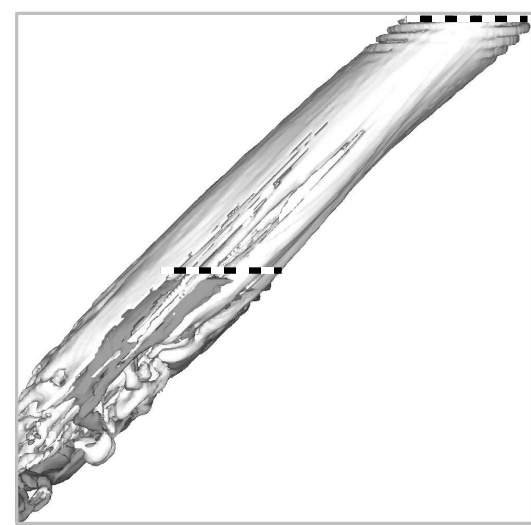

c) Yaw angle $=45^{\circ}$

Figure 9. Wake developed by the reference and downwind rotors when operating at three different angles of yaw to the relative wind $\left(x_{h}=4 \mathrm{R} ; y_{h}=4 \mathrm{R} ; \lambda=8\right)$.

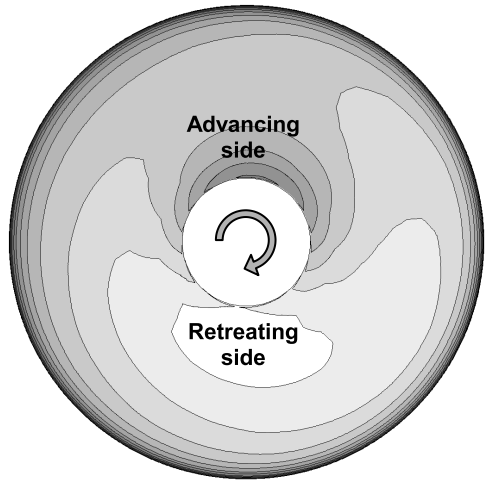

a) Yaw angle $=15^{\circ}$

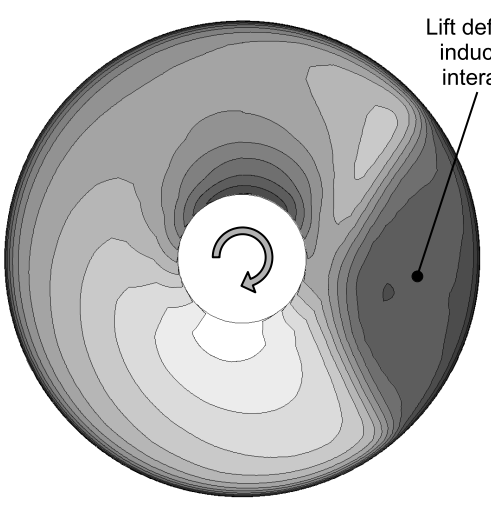

b) Yaw angle $=30^{\circ}$
$\mathrm{C}_{\mathrm{n}}$

0.500

0.464

0.429

0.393

0.357

0.321

0.286

0.250

0.214

0.179

0.143

0.107

0.071

0.036

0.000

c) Yaw angle $=45^{\circ}$

Figure 10. Distribution of blade normal force coefficient on the downwind rotor, during one complete revolution, when operated at three different angles of yaw to the relative wind (viewed from the downwind side of the rotor).

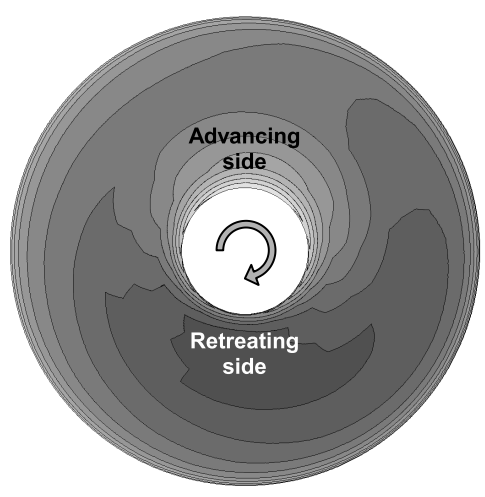

a) Yaw angle $=15^{\circ}$

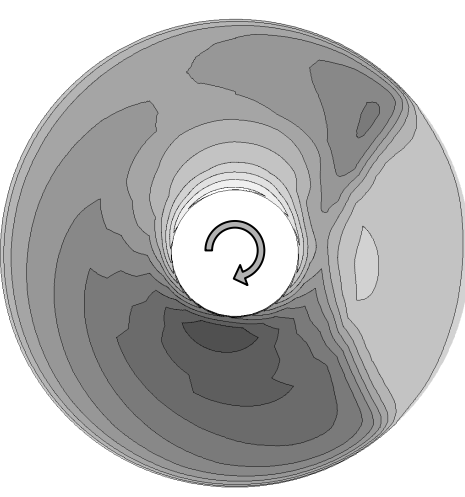

b) Yaw angle $=30^{\circ}$

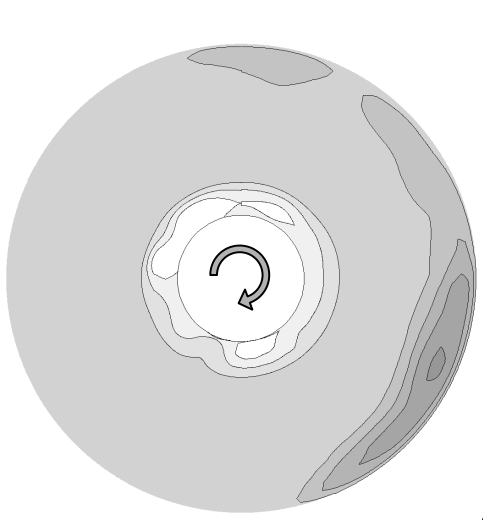

c) Yaw angle $=45^{\circ}$
$\mathrm{C}_{\mathrm{t}}$ 0.010 0.007

0.003

0.000
-0.003

$-0.007$

$-0.010$

$-0.013$

$-0.017$

$-0.020$

$-0.023$

$-0.027$

$-0.030$

$-0.033$

$-0.037$

(driving force)

Figure 11. Distribution of blade tangential force coefficient on the downwind rotor, during one complete revolution, when operated at three different angles of yaw to the relative wind (viewed from the downwind side of the rotor). A negative value of $\mathrm{C}_{t}$ represents a driving force on the rotor. 
The distribution of normal and tangential force coefficients on the downwind rotor are shown in figures 10 and 11 respectively, for each of the three angles of yaw simulated here. Figure 10(b) clearly illustrates the asymmetry in the normal force coefficient on the disk as the downwind rotor is partially immersed within the wake of the reference rotor. A large region of low aerodynamic loading is present on the windward half of the rotor disk, whilst a region of higher loading is found on the leeward half of the rotor disk. As such, an asymmetry in the aerodynamic loading is induced on the downwind rotor as a result of its interaction with the wake of the reference rotor. This azimuthal variation in aerodynamic loading as a result of interaction forms in addition to that created by the edgewise component of the wind velocity. Similar asymmetry may be observed in the distribution of tangential force over the disk of the downwind rotor, as shown in figure 11(b). In the rotor configurations simulated in this paper, the angle of attack is typically less than ten degrees at each of the radial stations along the blades. As a result, the negative tangential force that may be observed in figures $11(\mathrm{a})$ and $11(\mathrm{~b})$ over much of the downwind rotor provides a significant component of the torque that drives the rotor. Indeed, even though the magnitude of the normal force coefficient is, in general, significantly greater than that of the tangential force, only a small component of the normal force acts to drive the rotation of the rotor.

The relative locations of the two rotors is such that when the yaw angle is $45^{\circ}$, the wake of the reference rotor convects almost directly toward the downwind rotor. The geometry of the system therefore results in the direct impingement of the wake of the reference rotor on the downwind rotor, as shown in figure 9(c). As a result, the mean power coefficient of the downwind rotor is reduced to approximately $30 \%$ of that developed by the reference rotor, as shown in figure 8 . The significant reduction in the mean power coefficient that is observed in figure 8 correlates with the low values of normal and tangential force coefficient over the majority of the downwind rotor, as shown in figures 10 (c) and 11(c) respectively. Whilst some unsteadiness is evident in the performance of the downwind rotor when operating at a $45^{\circ}$ angle of yaw, the peak-to-peak amplitude of the fluctuations in power coefficient is lower than that when the rotors operate at $30^{\circ}$ angle of yaw. In practice, the unsteadiness in the loading on the blades would be detrimental to the fatigue life of the turbine. The results presented in figures 4 and 8 indicate that, of the three different inter-rotor separations simulated here, the unsteadiness resulting from aerodynamic interaction is most significant when the separation between the rotors is largest (in the current work, twelve rotor radii). Furthermore, the unsteadiness in performance is exacerbated when the rotors operate in yawed wind conditions such that the downwind rotor is partially immersed within the wake induced by the reference rotor. These findings are of significant practical interest given that the turbines operating within wind farms are typically separated by twelve or more rotor radii, and operate in yawed conditions during a large proportion of their useful life. It should be noted, however, that the unsteadiness in the power coefficient of the downwind rotor cannot continue to increase indefinitely for rotor separations greater than those simulated for this paper. Indeed, the breakdown of structure within the wake as a result of its natural instability will ensure that a separation distance will be reached beyond which the effect of the reference rotor on the downwind rotor will be negligible.

\section{The Effect of the Sense of Rotor Rotation}

It has been known for many decades that in certain flight conditions the performance of the tail rotor of a helicopter with a single main rotor is highly sensitive to the sense of its rotation. This effect is most certainly a result of aerodynamic coupling between the main and tail rotors, but for many years was not well understood. Recent studies have shown that the sensitivity of the performance of the tail rotor to its direction of rotation is related to the velocity distribution that is induced on the tail rotor by the vortical structures within the wake of the main rotor. ${ }^{15}$ These studies raise the question of whether the performance of a wind turbine may also be sensitive to its sense of rotation when it is aerodynamically coupled to the other turbines within a wind farm.

Figure 12 shows a comparison of the radial distributions of normal force coefficient and angle of attack as predicted by the VTM on rotors with opposing senses of rotation as they interact with a reference rotor mounted upstream. In the first case the downwind rotor rotates in the same sense as the reference rotor, whilst in the second case, the downwind rotor rotates in the opposite sense to the reference rotor. The spacing between the rotors is four rotor radii and the tip speed ratio is six for both of the configurations shown in the figure. Figure 12(a) clearly shows that when the sense of rotation of the downwind rotor opposes that of the reference rotor, the downwind rotor develops a lower normal force coefficient over the inboard portion of the blade when compared to the downwind rotor that has the same sense of rotation as 


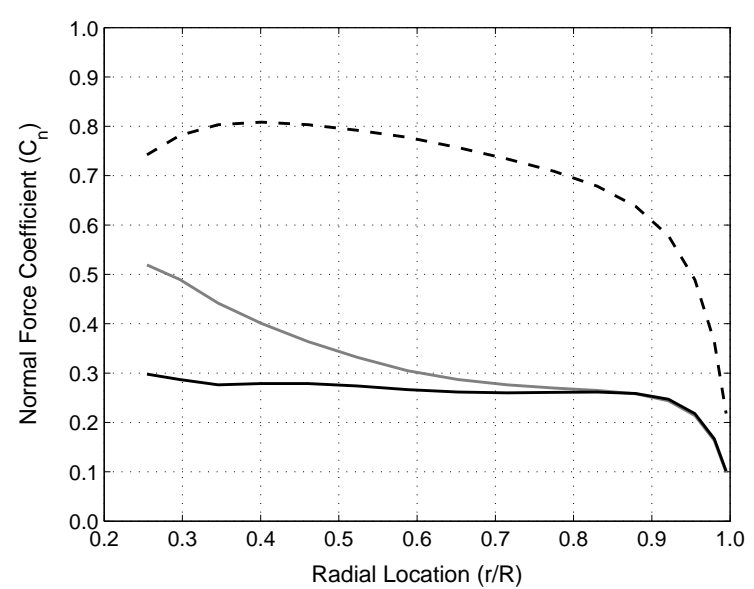

a) Radial distribution of normal force coefficient.

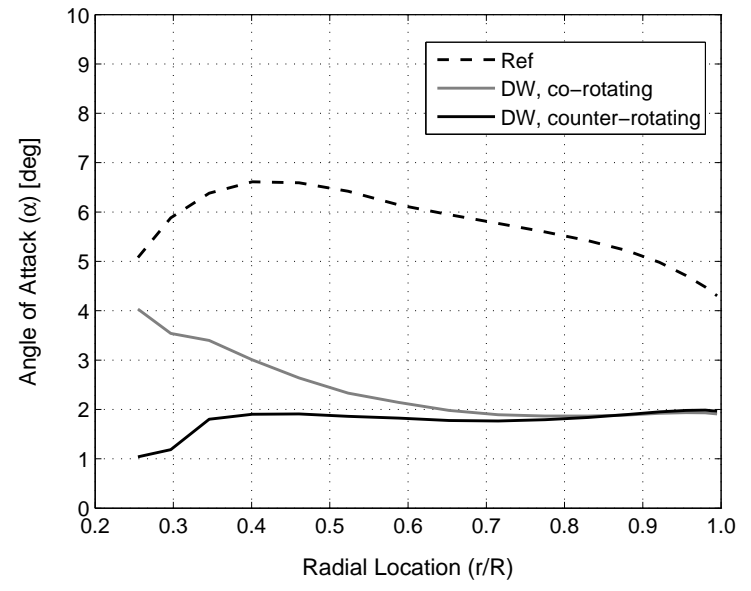

b) Radial distribution of angle of attack.

Figure 12. Effect of sense of rotation on the distribution of normal force coefficient and angle of attack along the blades of the downwind rotor; $\lambda=6$ (Ref: reference rotor, DW: downwind rotor).

the reference rotor. The reduction in force developed on the blades correlates directly with a lower angle of attack along a significant portion of the blades, as shown in figure 12(b).

Despite the marked differences in the performance of the blades shown in figure 12, reversing the sense of rotation of the downwind rotor has only a very small effect on the power coefficient that it develops. The quasi-steady power coefficient of the downwind turbine that has the same sense of rotation to the reference rotor is 0.117 compared with 0.109 for the downwind turbine with the opposite sense of rotation. It should be remembered, however, that the relationship between the aerodynamic loading on particular portions of the blades and the power coefficient of the rotor is far from straightforward. Indeed, the rotor is driven only by those blade sections at which the forward component of the lift is large enough to overcome the profile drag, and therefore where the rotor absorbs energy from the wind. Figure 12(a) suggests, nevertheless, that the loads that are developed on aerodynamically coupled turbine rotors within wind farms can be, to some extent, influenced by the relative sense of rotation of the turbine rotors. It should be borne in mind, however, that the current turbine model does not account for the presence of a hub, transmission assembly, or tower. In practice, the vortices trailed from the roots of the blades would possess neither the same strength or coherence as those computed in the present study. Instead, a highly unsteady and disordered wake would form downwind of the multiple elements that compose the rotor hub. These added complications may have significant effect on the results presented in figure 12 .

\section{Conclusion}

The aerodynamic interactions between wind turbines in both axial and yawed wind conditions has been simulated in order to better understand how the power coefficient of wind turbine rotors is influenced by the wakes generated by rotors located further upwind. The dependence of the power output of a wind turbine rotor that is subject to aerodynamic interaction with the wake of another turbine on tip speed ratio, the separation between the rotors, the angle of yaw to the incident wind, and the sense of rotation of the downwind rotor with respect to the rotor located further upwind has been investigated.

A rotor located downwind of another suffers a reduction in its aerodynamic power output if subject to impingement by the wake of the upstream rotor. Less power is lost as the separation between the rotors is increased, but the simulations performed in this paper suggest that rotors located even twelve radii apart may experience a $40-50 \%$ reduction in power for tip speed ratios in the range $6-8$. The power coefficient developed by the downwind rotor, as a fraction of its performance in an undisturbed wind, reduces as the tip speed ratio of the rotors increases. In yawed wind conditions, the largest reduction in the mean power coefficient occurs when the wake of the upstream turbine rotor impinges on the entire rotor disk. Considerable unsteadiness can be induced in the performance of the downwind rotor, however, when the yaw angle is such that the rotor disk is partially immersed within the wake of the upstream rotor. This unsteadiness in performance results 
from the asymmetry that is created in the normal and tangential components of force on the blades when subject to a highly asymmetric interaction with the wake induced by the upstream rotor. The aerodynamic loading on the inboard portion of the blades of a wind turbine rotor interacting with another rotor that is located upwind, appears, to some extent, to be sensitive to the direction in which the downwind rotor rotates with respect to the upwind rotor. The evolution of the wake of the upstream turbine plays a critical role in governing the power that is developed by rotors located further downwind. The primary effect of interaction with the wake of the upstream rotor is to reduce the local wind speed at the downstream rotor. Crucially, though, the natural instability of the turbine wake acts to moderate the deficit in wind speed at the downwind rotor and, as the distance between the rotors is increased, the breakdown of structure within the wake of the upstream rotor allows a recovery in the performance of the downwind rotor.

The separation distances between the rotors simulated in the present work are similar to, or somewhat smaller than those currently found in many wind farms. The results presented in this paper indicate particularly the adverse effects on the performance of a wind farm that may be expected by increasing the density of its constituent turbines. Indeed, the growth of the wind energy industry, particularly in terms of onshore developments, will be accompanied by increasing constraints on the availability of space for turbines and will therefore rely increasingly on the efficient utilization of suitable sites. As such, it is hoped that the numerical techniques and results described in this paper will be of use to the developers of wind farms in helping to reduce the inefficiencies that arise from aerodynamic interactions between the individual turbines that constitute the farm.

\section{References}

${ }^{1}$ Vermeer, L.J., Sørensen, J.N., and Crespo, A., "Wind Turbine Wake Aerodynamics," Progress in Aerospace Sciences, Vol. 39, No. 6-7, 2003, pp. 467-510.

${ }^{2}$ Crespo, A., Hernández, J., and Frandsen, S., "Survey of Modelling Methods for Wind Turbine Wakes and Wind Farms," Wind Energy, Vol. 2, No. 1, 1999, pp. 1-24.

${ }^{3}$ Newman, B.G., "The Spacing of Wind Turbines in Large Arrays," Energy Conservation, Vol. 16, No. 4, 1977, pp. $169-171$.

${ }^{4}$ Builtjes, P.J.H., and Smit, J., "Calculation of Wake Effects in Wind Turbine Parks," Wind Engineering, Vol. 2, No. 3, 1978, pp. 135-145.

${ }^{5}$ Lissaman, P.B.S., "Energy Effectiveness of Arbitrary Arrays of Wind Turbines," Journal of Energy, Vol. 3, No. 6, 1979, pp. $323-328$.

${ }^{6}$ Milborrow, D.J., "The Performance of Arrays of Wind Turbines," Journal of Wind Engineering and Industrial Aerodynamics, Vol. 5, No. 3-4, 1980, pp. 403-430.

${ }^{7}$ Sforza, P.M, Sheering, P., and Smorto, M., "Three-dimensional Wakes of Simulated Wind Turbines," AIAA Journal, Vol. 19., No. 9, 1981, pp. 1101-1107.

${ }^{8}$ Ainslie, J.F., and Hassan, U., Parkinson, H.G., and Taylor, G.J., "A Wind Tunnel Investigation of the Wake Structure within Small Wind Turbine Farms," Wind Engineering, Vol. 14, No. 4, 1990, pp. 24-28.

${ }^{9}$ Voutsinas, S.G., Rados, K.G., and Zervos, A., "On the Analysis of Wake Effects in Wind Farms," Wind Engineering, Vol. 14, No. 4, 1990, pp. 204-219.

${ }^{10}$ Barthelmie, R.J., Frandsen, S.T., Rathmann, O., Hansen, K., Politis, E.S., Prospathopoulos, J., Cabezon, D., Rados, K., van der Pijl, S.P., Schepers, J.G., Schelez, W., Phillips, J., and Neubert, A., "Flow and Wakes in Large Wind Farms in Complex Terrain and Offshore," European Wind Energy Conference, Brussels, Belgium, 31 March - 3 April, 2008.

${ }^{11}$ Brown, R.E., "Rotor Wake Modeling for Flight Dynamic Simulation of Helicopters," AIAA Journal, Vol. 38, No. 1, 2000, pp. $57-63$.

${ }^{12}$ Brown, R.E., and Line, A.J., "Efficient High-Resolution Wake Modeling using the Vorticity Transport Equation," AIAA Journal, Vol. 43, No. 7, 2005, pp. 1434-1443.

${ }^{13}$ Weissinger, J., "The Lift Distribution of Swept-Back Wings, NACA Technical Memorandum," No. 1120 , March 1947.

${ }^{14}$ Toro, E.F, "A Weighted Average Flux Method for Hyperbolic Conservation Laws," Proceedings of the Royal Society of London, Series A: Mathematical and Physical Sciences, Vol. 423, No. 1865, 1989, pp. 401-418.

${ }^{15}$ Fletcher, T.M., and Brown, R.E., "Main Rotor - Tail Rotor Interaction and its Implications for Helicopter Directional Control," Journal of the American Helicopter Society, Vol. 53, No. 2, 2008, pp. 125-138.

${ }^{16}$ Kenyon, A.R., and Brown, R.E., "Wake Dynamics and Rotor-Fuselage Aerodynamic Interactions," Proceedings of the American Helicopter Society 63 ${ }^{\text {rd }}$ Annual Forum, Virginia Beach, Virginia, USA, 1-3 May 2007.

${ }^{17}$ Green, R.B., Gillies, E.A., and Brown, R.E., "The Flow Field around a Rotor in Axial Descent," Journal of Fluid Mechanics, Vol. 543, No. 1, 2005, pp. 237-261.

${ }^{18}$ Ahlin, G.A., and Brown, R.E., "The Vortex Dynamics of the Rotor Vortex Ring Phenomenon," Proceedings of the 63" American Helicopter Society Annual Forum, Virginia Beach, Virginia, USA, 1-3 May 2007.

${ }^{19}$ Kim, H.W., and Brown, R.E., "Coaxial Rotor Performance and Wake Dynamics in Steady and Manoeuvring," Proceedings of the 62 $2^{\text {nd }}$ American Helicopter Society Annual Forum, Phoenix, Arizona, USA, 9-11 May 2006.

${ }^{20}$ Hand, M.M., Simms, D.A., Fingersh, L.J., Jager, D.W., Cotrell, J.R., Schreck, S., and Larwood, S.M., "Unsteady Aerodynamics Experiment Phase VI: Wind Tunnel Test Configurations and Available Data Campaigns," NREL Report TP500-29955, 2001. 